\title{
Funding the new biologics - Introduction
}

\author{
Alan Barkun MD CM MSC
}

$I^{n}$ up to $20 \%$ of cases, Crohn's disease remains active despite conventional medical therapy. Disorders of this type are usually not fatal, but result in significant disability, and thereby incur disproportionately great indirect medical and other costs to the patient and his or her family. Because treatment options are limited and there is a desire to avoid repeated surgical procedures, new biological agents, such as infliximab, are welcomed by physicians and patients.

Infliximab is expensive, however, and a recent Canadian economic analysis has suggested that its use exceeds recognized benchmarks of cost effectiveness. This analysis was rigorous, used the best available clinical data, and included sophisticated sensitivity analyses, but was subject to important limitations dictated by the available data. For example, it assessed only direct medical costs and employed a short time horizon, which might have resulted in an underestimation of the long term benefits of averting surgery. Some of the assumptions that were used in the design of the model relied on American epidemiological data and expert opinion, instead of empirical Canadian data.

The use of quality-adjusted life-years in the calculation of clinical costs and benefits might not be appropriate for patients with relatively high indirect medical costs, as is the case with Crohn's disease. It might be better to compare the benefits of a specific medical strategy with those obtained by alternative uses of the health care resources. The manner by which quality-adjusted life-years were calculated in the Canadian Coordinating Office for Health Technology Assessment report has also been discussed.

Currently accepted standards of cost effectiveness, including incremental cost utility ratios, are inherently arbitrary. It is possible that, in general, new biological agents could never meet such standards and would thus be declared cost ineffective, despite the fact that they are of proven benefit for certain important subgroups of patients. It has been suggested that a new way of evaluating these agents must be devised. Because the cost of the medication itself is an important component of overall health care costs, innovative cost-sharing schemes should be considered. It would also be helpful to identify specific subgroups of patients for whom new and expensive agents are appropriate.

Clinical experience with infliximab is limited, and further economic analyses will be required as new data become available. Policy makers will need to consider not only economic but also humanitarian factors when deciding whether to provide funding for medications that offer hope to patients whose prognoses are otherwise dismal.

The following papers were presented at a workshop held at the University of Calgary on September 24, 2002. The workshop was supported by an unrestricted grant from Schering Pharmaceuticals. It was organized by the Institute of Health Economics and the Lloyd Sutherland Group. 


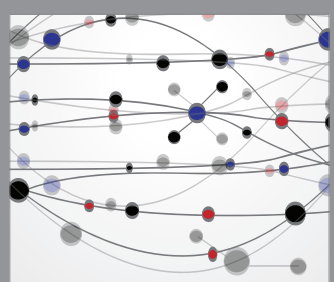

The Scientific World Journal
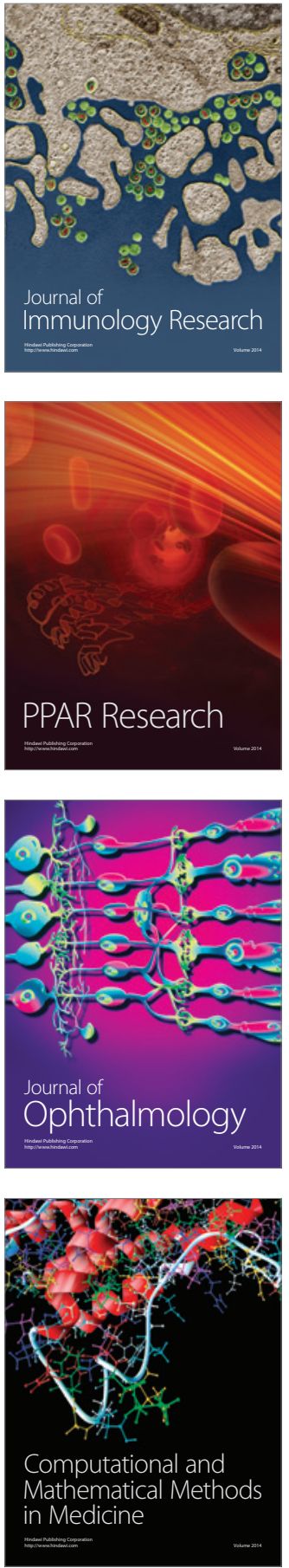

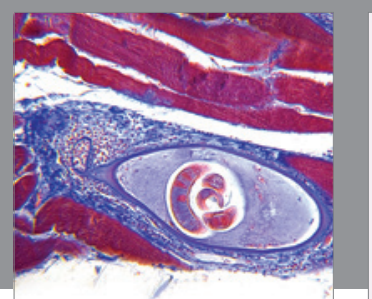

Gastroenterology Research and Practice

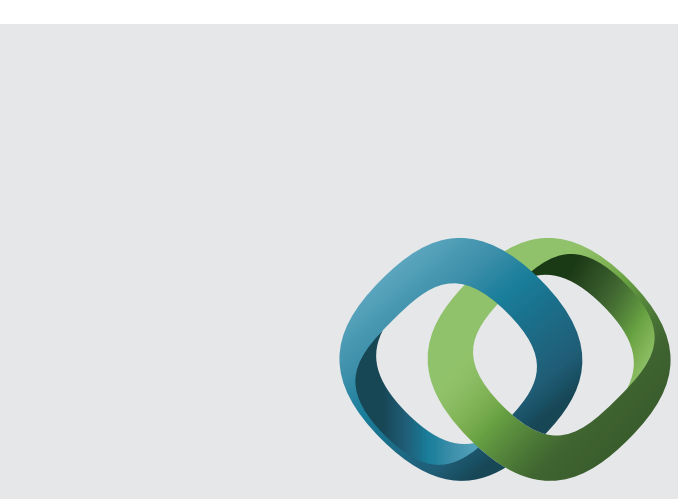

\section{Hindawi}

Submit your manuscripts at

http://www.hindawi.com
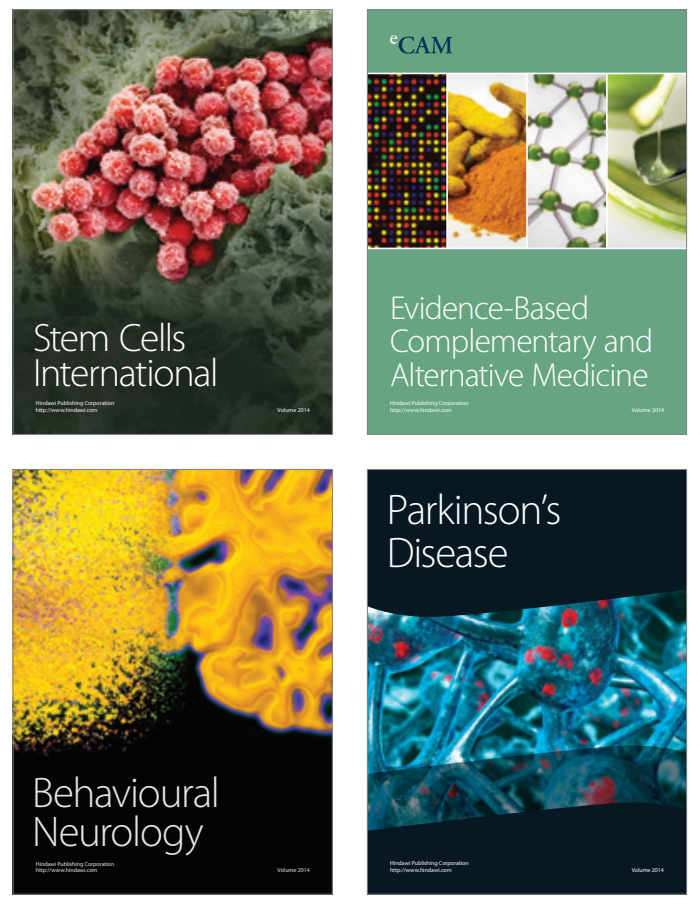
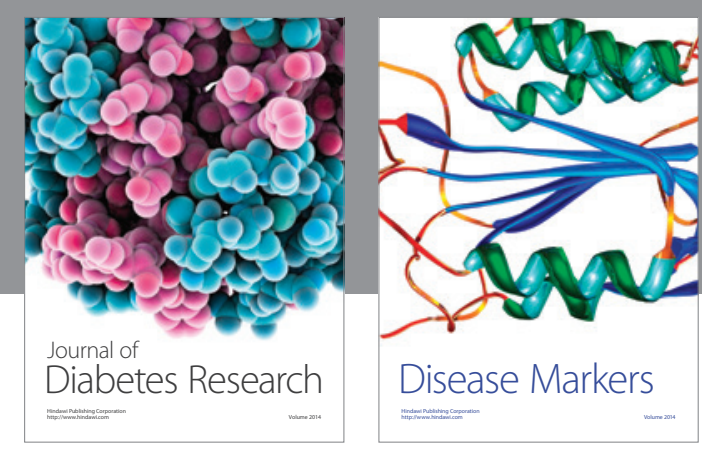

Disease Markers
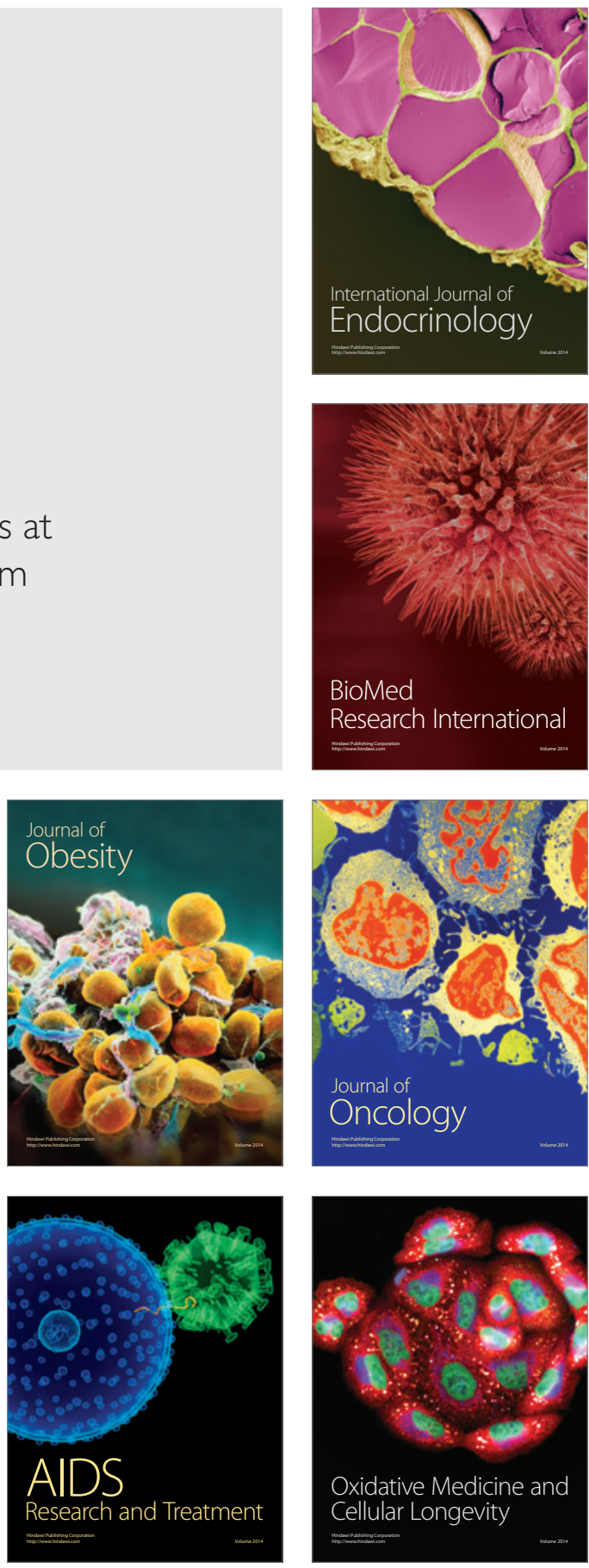